(Aus der chirurgischen Klinik und dem pathologischen Institut der Akademie für praktische Medizin zu Düsseldorf.)

\title{
Über ein primäres Rundzellensarkom beider Nieren bei einem Kinde.
}

\author{
Von Dr. Henri Schwers und Dr. Albrecht Wagner.
}

(Mit 8 Abbildungen im Text.)

Aus der akademisch-chirurgischen Klinik zu Düsseldorf (Direktor : Geh. Rat Professor O. Witzel).

\section{Klinischer Teil.}

Von Dr. Henri Schwers (aus Lüttich), Assistent der Klinik.

Am 12. II. IgI3 wurde der chirurgischen Kinderabteilung ein 8 jähriges Mädchen, K. B., mit der Diagnose tuberkulöse Bauchfellentzündung zugeschickt; ich stellte aber sofort die Diagnose: doppelscitiger Nierentumor, und zwar Cystennicren. Unter dieser Diagnose wurde das Kind bis zu seinem Tode an Bronchopneumonie mit Mittclohrentzündung am 3. X. 1913, etwa 8 Monate lang, auf der Klinik geführt. Da mir die Diagnose auch weiterhin stets sicher zu sein schien, bot es cine gewisse Überraschung, als die von Herm Professor Mönckeberg ausgeführte Sektion außer beiderseitigen Geschwülsten Verschleppungen in den retroperitonealen, trachealen und linkseitigen supraclavicularen Drüsen ergab, und daß sich mikroskopisch die Geschwulst als rundzelliges Nierensarkom erwies. Da Fälle von doppeltem primären Nierensarkom äußerst selten sind (4 Beobachtungen beim Menschen, I beim Hund) und kaum klinisch so gut verfolgt werden konnten wie der vorliegende Fall, möchte ich die ganze Krankengeschichte wiedergeben, was um so mehr Interesse hat, als das Kind aus anderen Gründen schon das Jahr zuvor in den hiesigen Krankenanstalten beobachtet wurde, und 
zwar auf der Kinderklinik von Professor Schloßmann, der mir freundlichst die damaligen Befunde mitteilte.

K. B., geboren am 17. I. 1905, hatte eine gesunde, doch etwas bleichsüchtige Mutter; der Vater hustete seit ro Jahren; es hatte nur eine Schwester, welche an Rückgratverkrümmung litt.

Beginn 1912 hatte die Patientin Masern und kam darauf wegen Verdacht auf Kcuchhusten und Brusthöhlenempyem zur Infektionsabteilung der Kinderklinik. Am 28. IV. 1912 wegen eines linkseitigen Brusthöhlenergusses nach der Kinderklinik verlegt, wurde es dort am 29. IV. I9I2 thorakotomiert. Es ergab sich cin großes, rein seröses Exsudat mit Lympho- und Leukocyten, ohne Bakterien (später wurden zahlreiche grampositive Diplokokken nachgewiesen). Am I. V. 1912 stieg plötzlich bei dem vorher fieberfreien Kinde die Temperatur auf 40 Grad und schwankte um 39 Grad bis zum 6. V. IgI2. Da crkannte man, daß das Kind an Scharlach erkrankt sei, und es wurde wieder zur Infektionsabteilung der Kinderklinik verlegt. Am 8. VII. I9I 2 war das Kind von seinem Scharlach geheilt, und kam zur Erholung zur Kinderklinik zurück, die es am 17. VII. I9I2 geheilt entlieb.

Aus vorstehenden Daten ergibt sich, daß das Kind im Jahre 1912, innerhalb Jahresfrist vor dom Bestehen großer Nierentumoren, Infektionskrankheiten durchmachte, und da $\beta$ es drei Monate lang, vom siebenten bis zum zehnten Monat, bevor die Nierentumoren

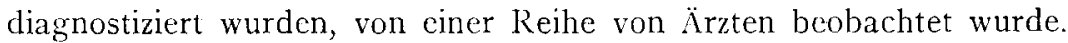
Während dieser Zeit ergab die Untersuchung keinen palpablen Nierentumor. Am 8. VII. I9I2 ist ,das Abdomen sehr breit, weich, im Thoraxniveau, die Leber zwei Finger breit unter dem Rippenniveau, die Milz nicht palpabel". Auch waren die Nieren durch den Scharlach anscheinend nicht besonders angegriffen, da die Eiweißprobe im Urin fast stets negativ ausfiel. Das Allgemeinbefinden war gut: Körperlänge $104 \mathrm{~cm}$, Kopfumfang $5 \mathrm{I} \mathrm{cm}$, Brustumfang $55-56 \mathrm{~cm}$, Gewicht 18, I kg; leichte Skoliose. Tuberkulineinspritzungen von I ccm. $1 / 1000$ und $1 / 100$ blicben ohne Reaktion. Am 17. VII. 19I2 „, sieht das Kind sehr wohl aus, hustet noch wenig“ und wird geheilt von der Kinderklinik entlassen.

Sieben Monatc später kommt das Kind zu den Krankenanstalten zurück und wird in die chirurgische Klinik aufgenommen. Der von mir beobachtete Aufnahmebefund am 12. II. 1913 war der folgende.

Das nun 8jährige Kind hat ein altes Gesicht, ist ziemlich mager und blaß; dennoch ist die Tuberkulinreaktion nach von Pirquet negativ. Die Patientin ist fieberfrei. Der Bauch ist auffällig und regelmäßig aufgctricben und hat cinen Umfang von etwa $75 \mathrm{~cm}$. In der Bauchhöhle ist keine freie Flüssigkeit vorhanden, dagegen sind rechts und links im Abdomen zwci riesige, etwa $20.20 \mathrm{~cm}$ rechts, $22.15 \mathrm{~cm}$ links messende feste Tumoren, die nach Lage und 
Form den beiden Nieren entsprechen. Die Oberfläche dieser Geschwülste ist leicht unregelnäßig; die rechte trägr zwei fast haselnußgroße Cysten; zwischen den beiden Tumoren ist nichts zu crmitteln, auch nicht im Bade; nur in Narkose ist eine kleine Verdickung und Verhärtung in der Tiefe der Pylorusggegend zu fühlen. Das Röntgenbild ergibt den Tumoren entsprechenden Schatten, doch bringt es nichts Neues hinzu. Der Urin ist klar, alkalisch; die tägliche Menge schwankt um $1000 \mathrm{ccm}$, das spezifische Gewicht ist durchschnittlich Ior4; am Aufnahmetag betrug der Gefrierpunkt des Urins ( $(\Delta)$ -.. o,84 Grad, und die Harmstoffmenge ( $-U$ ) I, I6 Proz., letzteres ciner täglichen Harnstoffmenge von II,5g entsprechend; es war weder Eiweib noch Blut vorhanden.

Da bei rinem Mïdchen von 8 -ro Jahren nach Camerer (P f a n d I e r und Sch I o 13 mann, Handbuch der Kinderheilkunde, Bd. I, S. $266-267$ ) normalerweise dic Urinmenge $980 \mathrm{ccm}$ bei cinem spezifischen Ciewicht von nor6 beträgt, ferner die tïgliche Harnstoffmenge sich auf 14,7 beläuft, lronnte man von einer leicht herabgesetzten Nierenfunktion sprechen, aber nur, was die Menge der gelösten Bestandteile betrifft.

Das klinische Bild war also kurz das folgende: Einige Monate nach überstandenen Infektionskrankheiten (Masern, DiplokokkenPleuritis und Scharlach) Auftreten von beiderseitigen, groben, derben, cystisch erscheinenden Nierengeschwülsten, wclches von einer leichten Herabsetzung der Nierenfunktion begleitct war. Dieser Symptomkomplex sprach für Cystennieren. Andere Differentialdiagnosen konnten nach dem klinischen Befund nicht in Betracht kommen.

Wic schon mitgeteilt, erwies sich die Diagnose als nicht richtig, denn es lagen Nicrensarkome vor. Ehe ich an der Hand des Falles retrospektiv die Differentialdiagnose zwischen doppelter Cystenniere und doppeltem Nierensarkom bespreche, möchte ich den Verlauf der Erkrankung bei diesem Mädchen schildern.

Hervorhcben möchte ich zunächst, daß3 der Verlauf der Erkrankung zwei Perioden zeigte. In der crsten, etwa bis Juli IgI 3 , kämpfte der Organismus gegen die immer größer werdenden Tumoren und ihre liolgeerscheinungen; ab Juli I9 13 scheint der Organismus den Kampf als aussichtslos aufgegeben zu haben; er zerfällt, und mit ihm gehen auch dic Tumoren etwas zurück, sowie ein Teil der Begleiterscheinungen, während alle Symptome der Kachexic in den Vordergrund treten.

Schon in den Resultaten der wöchentlichen Gewichtsbestim- 
60 Schwers u. WagNer, C̈ber ein primäres Rundzellensarkom beider Nieren usw.

mungen des Kindes treten diese zwei Perioden hervor. Wie aus folgender Tabelle ersichtlich, ist das Gewicht innerhalb von 5 Monaten, bis Mitte Juli, selbst bis Mitte August gestiegen, und zwar von 22,5 auf $30 \mathrm{~kg}$ (mit Kleidern); dann aber geht das Gewicht mächtig zurück, so daß es Ende September nur noch $19,8 \mathrm{~kg}$ (ohne Kleider) beträgt.

Gewichtstabelle.

Datum Gewicht Bemerkung Datum Gewicht Bemerkung in $\mathrm{kg}$

\begin{tabular}{|c|c|c|c|c|c|c|c|}
\hline 4. II. 1913 & 20,6 & ohne & Kleider & 7. VI. I9I3 & 25,7 & mit & Kleidern \\
\hline I8. II. 19I3 & 21,0 & ", & $"$ & 14. VI. 1913 & 26,5 & , & ", \\
\hline I. III. $19 \mathrm{~J} 3$ & 21,6 & $"$ & $"$ & 2I. VI. I9I3 & 26,5 & $"$ & $"$ \\
\hline 8. III. 1913 & 22,2 & " & ", & 28. VI. 1913 & 27,6 & ” & $"$ \\
\hline I5. III. 1913 & 22,5 & mit & Kleidern & 5. VII. 1913 & $28, \mathrm{I}$ & ", & " \\
\hline 22. III. 1913 & 23,6 & ", & $"$ & $15 . \mathrm{VII} .1913$ & 27,5 & $"$ & $"$ \\
\hline 29. III. 1913 & 22,9 & $"$ & $"$ & 26. VII. IgI 3 & 29,0 & " & $"$ \\
\hline 5. IV. 1913 & $23, I$ & " & $"$ & 2.VIII. IgI 3 & 28,7 & , & $"$ \\
\hline I2. IV. 1913 & $23, I$ & $"$ & $"$ & 9.VIII. 1913 & 29,7 & ", & ", \\
\hline 19. IV. 1913 & 22,5 & $"$ & "y & I6. VIII. 1913 & 30,0 & $"$ & " \\
\hline 26. IV. 1913 & 24,0 & ", & , & 23.VIII. 1913 & 28,0 & ", & 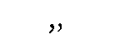 \\
\hline 3. V. 1913 & 22,9 & $"$ & " & 6. IX. 1913 & 22,0 & ohne & Kleider \\
\hline I7. V. 1913 & 24,0 & " & $"$ & 13. IX. 1913 & $2 \mathrm{I}, 5$ & , & , \\
\hline 23. V. I913 & 25,0 & ", & " & 20. IX. I9I3 & $2 \mathrm{I}, 5$ & $"$ & , \\
\hline o. V. I9I3 & 25,8 & ", & " & 27. IX, 19I3 & $\mathrm{I} 9,8$ & ” & $"$ \\
\hline
\end{tabular}

Nachdem das Gewicht bis Mitte Juli zugenommen, hat es nicht sofort, sondern erst nach Mitte August abgenommen, obgleich die Dekadenzerscheinungen schon Mitte Juli einsetzten. Dies ist wohl darauf zurückzuführen, daß die Tumoren, und besonders die retroperitonealen Drüsen, einen Monat gebraucht haben, um kleiner zu werden und so einen deutlichen Rückgang des Kompressionsödems der unteren Körperhälfte zu ermöglichen.

Besser noch als aus den Gewichtsangaben kann man das Allgemeinbefinden des Kindes nach den hier eingeschalteten Photographien erkennen, die im Beginn (kurz nach der Aufnahme und am 30. III. I913), zur Mitte (I8. VII. I913) und zum Ende (24. IX. I9I3) des Krankenhausaufenthalts aufgenommen wurden.

Es stelle sich bei der Patientin eine progressive Kachexie ein, die besonders stark in den drei letzten Monaten des Lebens (Juli bis September) war. Das Kind wurde immer matter, 


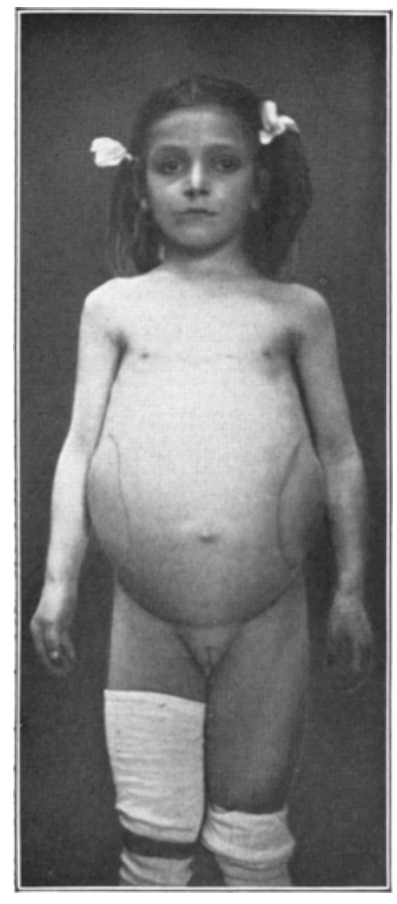

Fig. I. Februar I) 3.

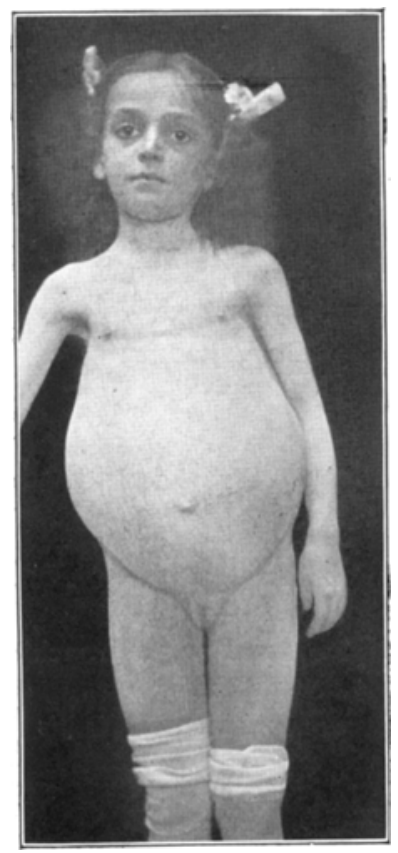

Fior 2 20 März I0I2.

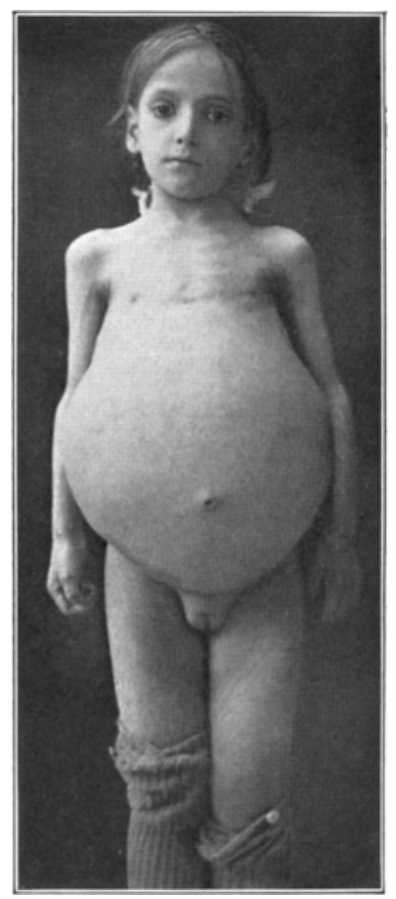

Iigr. 3. I8. Iuli ipr3.

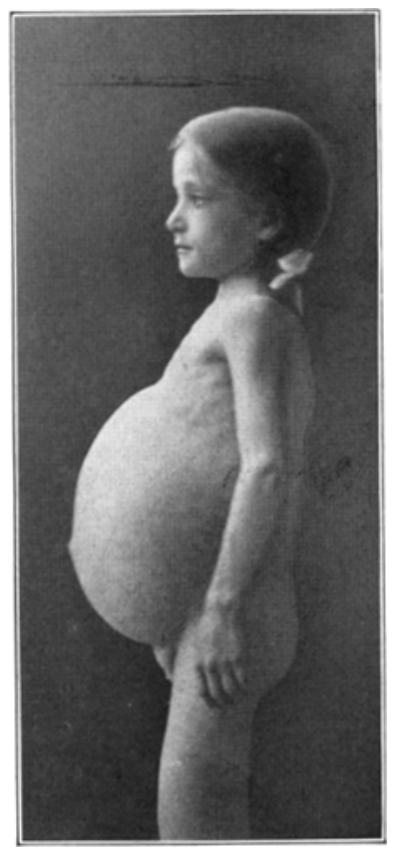

Fie. 4. I8. Iuli Iors. 
apathischer, blasser; i 4 Tage vor dem Tode war es kaum mehr imstande zu sitzen, und der Hämoglobingehalt des Blutes betrug nur noch I 5 Proz. (nach Gowers).

Die Erkrankung verlief fieberfrei, nur ausnahmsweise wurde am 9. IX. I9I $39,3^{\circ}$ verzeichnet, was wahrscheinlich auf Bronchitis und die damals beginnende Mittelohreiterung zurückzuführen war. Ferner traten bei der Bronchopneumonie der zwei letzten Lebenstage (mit Mittelohreiterung) auch hohe Temperaturen ein. Wie aus folgender Tabelle ersichtlich, lag cher bei den Rektalmessungen etwas Untertemperatur vor.

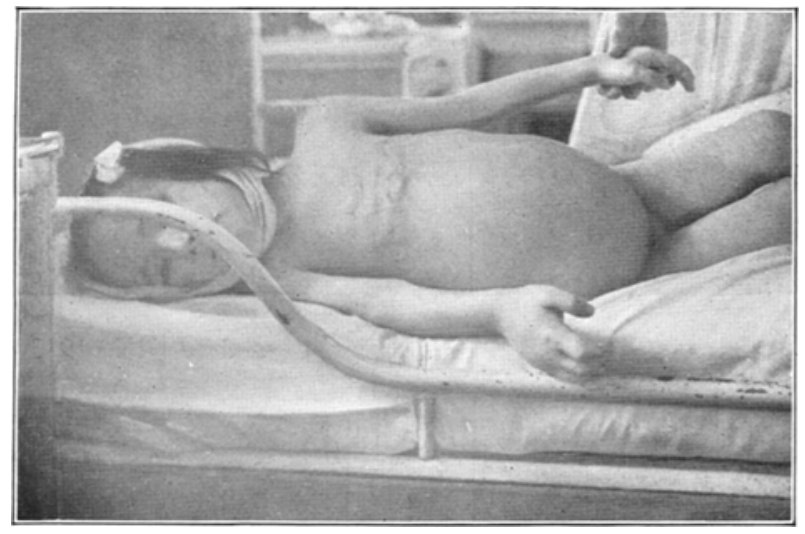

Fig. 5. 24. September I9I3.

Temperaturtabelle.

(Berechnete Durchschnittstemperaturen.)

\begin{tabular}{|c|c|c|c|}
\hline Monat & morgens & abends & Bemerkungen \\
\hline Februar & 37,2 & 37,3 & - \\
\hline März & 36,9 & $37, \mathbf{I}$ & - \\
\hline April & $37, \mathrm{I}$ & $37, \mathrm{o}$ & - \\
\hline Mai & 36,9 & 36,8 & - \\
\hline Juni & 36,7 & 36,7 & - \\
\hline Juli & 36,4 & 36,5 & Imal 38,0 \\
\hline August & $3^{6,7}$ & 37,0 & - \\
\hline \multirow[t]{3}{*}{ Oktober (2 Tage) } & $e^{37,0}$ & 37,4 & $\begin{array}{c}\text { Imal } 38,0 \text {, Imal } 39,3: \text { Bronchitis, } \\
\text { Otitis }\end{array}$ \\
\hline & 37,2 & 39,5 & Bronchopneumonie, Otitis \\
\hline & $37, \mathrm{I}$ & 39,6 & \\
\hline
\end{tabular}


Über ein primäres Rundzcllensarkom beider Nieren bei cinem Kinde. 63

zahl der Pulsfrequenz bei diesem 8- bis 9jährigen Mädchen die von Pfaundler und SchloBmann angegebene Zahl 89 betrachtet.

$$
\text { Pulstabelle. }
$$

(Maximum, Minimum und berechneter Durchschnittspuls.)

\begin{tabular}{|c|c|c|c|c|}
\hline Monat & Maximum & Minimum & Durchschnitt & Bemerkungen \\
\hline Februar & 106 & 90 & 95 & $\leftarrow$ \\
\hline März & 128 & 92 & 107 & - \\
\hline April & 136 & 86 & I I 4 & -- \\
\hline Mai & I 40 & 86 & 118 & - \\
\hline Juni & 128 & 88 & 106 & — \\
\hline Juli & I 32 & 100 & II 8 & - \\
\hline August & I 44 & 96 & I 18 & - \\
\hline September & 138 & 104 & II 6 & - \\
\hline Oktober ( $2 \mathrm{Tg}$. & I 44 & I I 8 & $13 \mathrm{I}$ & Bronchopneumonic, \\
\hline
\end{tabular}

Dicse hohe Pulsziffer kann wohl zum Teil durch das Verdrängen der Brustraumorgane durch die Bauchorgane, sowic auf dic linksseitigen Rippenfellverwachsungen zurückgeführt werden; zum Tcil kommt aber sicher auch dic Kachexie in Frage.

Als Begleiterschcinungen möchte ich hier Ödeme, Kopfschmerzen, Erbrechen und I)urchfälle erwähnen.

Die Ödeme beschränkten sich immer auf die unteren Gliedmaßen und die großen Labien des Kindes (siehe Photographien); bis Juli nahmen sie langsam zu, dann gingen sie bedeutend zurück. Nur ausnahmsweise kamen sie an einigen Tagen im Gesicht und an Händen auf, aber erst gegen Ende der Erkrankung.

Wiederholt, zu ganz unregelmäßigen Zeiten, klagte das Kind über Kopfschmerzen, oft tagelang, besonders aber in dem letzten Monat der Erkrankung.

Erbrechen trat unregelmäßig, vorwiegend in den letzten Monaten auf.

Im Februar und im März erbrach dic Patientin nicht.

Im April crbrach die Patientin $3 \mathrm{mal}$ an 3 Tagen.

Im Mai erbrach die Patientin nicht.

Im Juni erbrach die Patientin Imal.

Im Juli erbrach die Patientin $8 \mathrm{mal}$ an 7 Tagen.

Im August erbrach die Patientin gmal an 8 Tagen.

Im September erbrach die Patientin romal an 8 Tagen.

Durchfall wurde selten bcobachtet. Am 28. VIII. wurde $4 \mathrm{mal}$ Stuhl verzeichnet, ohne Urin, und am 24. bis 25 . IX. stellte sich der Durchfall abermals zwei Tage ein, doch mit Absonderung der gewöhnlichen IIarnmenge.

Der lokale Organbefund erwies folgende Veränderungen im Verlauf der Erkrankung.

Bis Juli 19I3 nahmen die Tumoren und damit der Bauchumfang 
stetig an Größe zu. Dann verkleinerten sich die Geschwülste und gleichzeitig; nahmen die ödeme der Beine und der äußeren Genitalion ab (siehe Abbildungen). Über die genauercn Maße gibt folgende kleine Skala Aufschluß.

Umfang des Bauches in der Höhe des Nabels

3o. III. 1913: $72 \mathrm{~cm}$

I3. V. IgI $3: 78 \mathrm{~cm}$

I8. IX. 1913:69 cm

Umfang des Oberschenkels ro $\mathrm{cm}$ oberhalb der Kniescheibe

8. VII. 19I3:30 $\mathrm{cm}$

18. IX. $1913: 23^{1 / 2} \mathrm{~cm}$

2. X. $1913: 2 \mathrm{I}^{1 / 2} \mathrm{~cm}$
Maximalumfang des Bauches (5-8 cm obcrhalb des Nabels)

3o. III. $1913: 77 \mathrm{~cm}$

8. VII. I9I3: $85 \mathrm{~cm}$

18. IX. $1913: 74 \mathrm{~cm}$

Umfang der Unterschenkel

(Wade)

8. VII. I9I $3: 24^{1 / 2} \mathrm{~cm}$

I8. IX. 1913: 181/2 $\mathrm{cm}$

2. X, 1913:17 cm

Die Harnmenge war, abgesehen vom letzten Monat der Krankheit, etwas gesteigert. Aber das spezifische Gewicht, der Gefrierpunkt und die Harnstoffmenge sprachen stets für herabgesetzte Nicrenfunktion. Jedoch sank die Tätigkeit der Nieren auch terminal kaum unter der Hälfte, wie aus folgenden Tabellen ersichtlich. Mit Camerer (loc. cit.) möchte ich als Normalwerte bei diesem 8--9jährigen Mädchen folgende annehmen:

Urinmenge: $980 \mathrm{ccm}$ täglich.

Sperifisches Gewicht: Io 6.

Harnstoffgehalt: $14,7 \mathrm{~g}$ täglich.

Dabei ist zu berücksichtigen, daß der Gefrierpunkt des Harns normalerweise zwischen - I,2 und -- I,7 Grad Celsius schwankt.

$$
\text { Urintabellen. }
$$

Monat TäglicheUrinmenge SpezifischesGewicht

(berechnete

Durchschnittsmenge)

Februar

März

April

Mai

Juni

Juli

August

September
$1000 \mathrm{ccml}$

$1330 \mathrm{ccm}$

$1200 \mathrm{ccm}$

$1360 \mathrm{ccm}$

$1080 \mathrm{ccm}$

$1000 \mathrm{ccm}$

$880 \mathrm{ccm}$
$1220 \mathrm{ccm}$ (berechnetes durchschnittliches spezifisches Gewicht)
IOI 4
1010
1008
1065
1008
1008
1007
I006
D a tum
12. II. 1913
Gefrierpunkt des Harns
Harnstoffmenge $+U$
$\triangle$, in Grad Celsius
Proz.
Gramm pro Tag
(berechnet)
8. V. I9I3
$-0,84$
$-0,58$
I, I 6
0,60
11,5
8,2 
Über ein primäres Rundzellensarkom beider Nieren bei einem Kinde. 65

Dat um Gefricrpunkt des Harns Harnstoffmenge $\wedge$, in Grad celsius I'roz. Gramm pro Tag (berechnet)

$\begin{array}{rrrr}\text { I4. VI. I9I3 } & -0,49 & 0,95 & 1 \mathrm{I}, 2 \\ \text { 4. VII. 19I3 } & -0,59 & \mathrm{I}, 04 & 10,4 \\ \text { 22. VII. I913 } & -0,74 & 1,13 & 11,3 \\ \text { I5. VIII. 1913 } & -0,54 & 0,58 & 7, \mathrm{I} \\ \text { 26. VIII. 1913 } & -0,5 \mathrm{I} & 0,86 & 10,5 \\ \text { II. IX. 1913 } & -0,47 & 0,60 & 5,3 \\ \text { 22. IX. 1913 } & -0,45 & 0,80 & 7,0\end{array}$

Letztere Bestimmungen von $\angle$. und -t- U wurden von Herrn Dr. Reinbach, Assistent des Herrn Professor M uller am hicsigen Biochemischen Institut, ausgeführt.

Der Urin blieb stets klar, Blut war nie sichtlich vorhanden. Dagegen fand sich von August ab EiweiB in geringen Mengen; am I8. IX. I913, I4 Tage vor dem 'Tode des Kindes, betrug dic Menge $3 / 4$ Proz. (nach Es bach).

Das Auftreten der Nierentumoren nach Infektionskrankheiten, die Doppelseitigkeit der Erkrankung, das Iiehlen von Fieber und Schmerz (nur Druck), das Vorhandensein leichter Verdauungsstörungen, die Erhaltung der Form der Nieren, die derbe Konsistenz und die Cysten derselben, die Erhaltung, selbst die Vermehrung der Urinmengen mit Herabsetzung des Gehaltes an gelösten Substanzen, dic Verminderung der Urinmenge nur in dem Endstadium, das Fehlen von Blut im Urin, und das späte Auftreten von geringen Mengen Eiweil3 im Harn, das progressive Abmagern, alle diese Symptome entsprachen dem Bilde der doppelseitigen Cystenniere. Gegen die Jiagnose Cystennicren sprach aber das Fehlen von jedem Nierenbefund bei der sorgfältigen Untersuchung der Kinderklinik am 8. VII. 19I2, und besonders das Fehlen der so häufigen, plötzlichen, periodischen Anfälle von akuter Urämie (siehe Paul Wagner, Verletzungen und chirurgische Isrkrankungen der Nieren, in Fisch und Zuckerkand1, Handbuch der Urologie, I3d. II, S. 330 u.f.). Die ödeme sind nur an den unteren Glicdern und an den Labien aufgetreten (ich sehe hier vom Gesichts- und Handödem des Terminalstadiums ab); sie haben mit den Tumoren zugenommen, sie sind mit dem Rückgang der Tumoren so gut wie verschwunden; es waren also reine Kompressionsödeme -- durch prävertebrale Drüsen, wie auch die Obduktion ergab -.. Die Kopf- 
schmerzen, die unregelmäßig und besonders terminal aufgetreten sind, können auf die Anämie und die Verdauungsstörungen zurückgeführt werden. Das Erbrechen war spärlich und kann durch die Kompression des Magens erklärt werden. Endlich sind die Durchfälle bis auf drei Tage ganz ausgeblieben. Diese Zeichen: Ödeme, Kopfschmerzen, Erbrechen und Durchfall haben also hier teils nicht den Wert von urämischen Erscheinungen, teils kamen sie nicht zum Vorschein. Sollten sie dennoch, soweit vorhanden, von anderer Seite als urämische Erscheinungen aufgefaßt werden, so hätten immerhin nicht schwere, akute, urämische Anfälle vorgelegen (nie Krämpfe, nie Ohnmacht), wie es bei Cystennieren der Fall ist, sondern die leichte, chronische Urämie, die als mögliche Begleiterscheinung der bösartigen Nierentumoren bekannt ist (siehe Steffen, Die malignen Tumoren im Kindesalter, S. 40).

Gegen die Diagnose Cystennieren sprachen aber noch verschiedene Zeichen, die auf eine bösartige Geschwulst hindeuteten. Das Wachstum der Tumoren war auffallend rasch; es stellte sich ein Rückgang der Geschwulst ein, der als Zerfall aufgefaßt werden konnte; es war in der Pylorusgegend eine prävertebrale Verdickung vorhanden, die als Drüsenmetastasen bezeichnet werden konnte; es war besonders zuletzt eine ausgesprochene Kachexic vorhanden, welche schlecht durch die Herabsetzung der Nierenfunktion allein erklärt werden konnte. In Berücksichtigung des fieberfreien Verlaufs war auch der Puls auffallend beschleunigt.

Nachdem Obduktion und mikroskopische Untersuchung ein beiderseitiges, primäres, rundzelliges Nierensarkom ergeben haben, mit Blutergüssen, die sich wie Cysten angefühlt hatten, mit Drüsenverschleppungen, die ja zum Teil gefühlt worden waren, doch nicht als solche erkannt, muß ich zugeben, daß trotz der Reihe positiver Elemente, welche für doppelte Cystenniere sprachen, genügend Zeichen da waren, um eine bösartige Geschwulst festzustellen, und zwar die eben aufgezählten, welche allerdings mehr aus dem Verlauf als aus einer einmaligen Befundaufnahme bei dieser Erkrankung sich ergeben.

Gelernt wird bei diesem Fall, daß die Differentialdiagnose: Cystenniere, primäres Nierensarkom nicht auf die bisher angeführten wichtigsten Zeichen von Cystennieren, wie Doppelseitig- 
Uber ein primäres Rundzellensarkom beider Nieren bei einem Kinde. 67

keit, Vorhandenscin von palpablen, cystischen Gebilden, Auftreten von Druckschmerz allein, beruhen darf. Will man sicher sagen, ob Cystennieren oder primäre Nierensarkome vorliegen, so müssen, neben den in beiden Fällen auftretenden Symptomen, wie Anämie, Abmagerung, einseitige oder beiderseitige große harte Tumoren mit Cysten, Kompressionsödeme, Druckschmerz, Erbrechen, Durchfall, Herabsetzung der Nierenfunktion, Fehlen von Blut im Harn, Vorhandensein von kleinen Mengen Eiweiß im Urin, a uch ganz typische Symptome vorliegen, welche nicht täuschen können, die sich meist aus dem Verlauf der Erkrankung ergeben: Bei Cystennieren wiederholte, akute, urämische Anfälle; bei primären Nicrensarkomen schnelles Wachstum der Geschwulst, Kachexie, Irüsenmetastasen, Verkleinerung der Tumoren durch Zerfall, auch Blut und Tumorpartikelchen im Urin.

Nach diesem Fall ist man also tatsächlich imstande, in vivo beiderseitige Cystennieren von beiderseitigen Nierensarkomen zu unterscheiden; man muß sich nur eine Beobachtungszcit lassen und bei der Diagnose nur die Symptome in Erwägung ziehen, die wirklich typisch sind; und das sind nicht mehr alle diejenigen, die bisher für klassisch galten.

Aus dem pathologischen Institut der Akademie für praktische Medizin (Direktor: Prof. J. G. Mönckeberg).

\section{Pathologisch-anatomischer Teil.}

Von Dr. Albrecht Wagner, Assistent des Institutes.

Am 4. X. I II 3 kam die Leiche des Kindes mit der klinischen Diagnose „Doppelscitige Cystennieren“ zur Obduktion. Die von Herrn Professor Mönckeberg ausgeführte Autopsie ergab folgendes:

Entsprechend großer und gracil gebauter weiblicher Körper in schlechtem Ernährungszustand mit stark vorgetriebenem Abdomen, dessen Decken aber nicht besonders stark gespannt erscheinen. Haut überall blaß, in dünncn Falten abhcbbar. Am Thorax links seitlich 